\title{
Effet protecteur des acides gras contre le stress oxydatif : Implication en physiopathologie vasculaire
}

Anne NEGRE-SALVAYRE

Robert SALVAYRE

Inserm U466 et laboratoire de biochimie, IFR31, CHU Rangueil, 31432, Toulouse, France
Article recu le 20 septembre 2005 Accepté le 9 novembre 2005

\begin{abstract}
Oxidative stress play a major role in the genesis and the evolution of vascular pathologies. Recent data suggest that redox mechanisms are involved in the signaling network of vascular cells and the physiology of the vascular wall. Experimental cell models allow to analyse the biological effect of pro-oxidants, and the regulation exerted by antioxidants and cytoprotective agents.

Fatty acids play a complex role in atherosclerosis, since they exhibit both anti and proatherogenic properties, as function of their concentration and insaturation level. Beside their role in cell metabolism and mitochondrial $\beta$-oxidation, fatty acids activate various signaling pathways, and could modulate intracellular oxidative stress via an uncoupling effect on mitochondria. We report here that fatty acids protect against mitochondrial oxidative stress by activating cellular glutathione peroxidase (GPX) activity. This effect is independent of the chain length and unsaturation level. EGF receptor is activated by fatty acids and involved in their protective effect against mitochondrial oxidative stress and GPX activation, and similar protective results are observed with EGF. Oleic acid and EGF protect DNA against the formation of 8oxodG lesions occurring subsequently to oxidative stress. Taken together, this new 'antioxidant' and cytoprotective property of fatty acids and EGF could participate to the stabilization of atherosclerotic plaque, and the more general anti-atherogenic properties of oleic acid.
\end{abstract}

Key words: fatty acids, EGF receptor, glutathione peroxidase, ROS oxidative stress, DNA lesion, 80xodG, apoptosis

\section{Intérêt général de l'étude}

Les processus d'oxydoréduction ont une fonction importante dans la signalisation des cellules vasculaires et la physiologie de la paroi artérielle, et jouent un rôle majeur dans la genèse des lésions d'athérosclérose et d'autres pathologies vasculaires. Les cellules vasculaires produisent des radicaux libres oxygénés (ROS) capables d'oxyder les acides gras polyinsaturés et d'initier les phénomènes de péroxydation lipidique (en particulier dans les LDL), et donc d'induire la formation des lésions primaires de la plaque d'athérosclérose. Par ailleurs, les cellules possèdent des systèmes antioxydants capables de neutraliser ou dégrader les ROS, et donc de limiter leurs effets délétères. Le rôle des acides gras dans ce domaine est mal connu. Les acides gras polyinsaturés sont une cible classique des ROS, mais ont également des propriétés antiathérogènes dont les mécanismes ne sont qu'imparfaitement connus. Nous faisons l'hypothèse que les acides gras pourraient sous certaines conditions, moduler la génération du stress oxydatif intracellulaire.

\section{Rappels sur le stress oxydatif en pathologie}

Les espèces réactives de l'oxygène (ROS) sont des dérivés de l'oxygène hautement réactifs et instables, participant au vieillissement des protéines, à la peroxydation lipidique, et à l'altération de I'ADN. Longtemps considérés comme des agents toxiques responsables de dysfonctions et de mort cellulaires, il est actuellement admis que les ROS sont de véritables seconds messagers impliqués dans l'expression de gènes, et la régulation des fonctions de prolifération et de mort cellulaire. De plus, ils sont des médiateurs inflammatoires impliqués dans diverses pathologies neurodégénératives ou vasculaires telles que l'athérosclérose ou l'hypertension [1, 2]. 


\section{Nature des ROS}

Les cellules génèrent divers types de ROS de réactivité différente [2-5] : - L'ion superoxyde $\mathrm{O}_{2}{ }^{\circ-}$ et le peroxyde d'hydrogène $\mathrm{H} 2 \mathrm{O} 2$ représentent une classe peu active. $\mathrm{O}_{2}{ }^{\circ-}$ peut interagir avec $\mathrm{H}+$ pour donner $\mathrm{HO}_{2}{ }^{\circ}$ qui serait la forme réactive de $\mathrm{O}_{2}{ }^{\circ-}$, capable d'initier la peroxydation lipidique. $\mathrm{O}_{2}{ }^{\circ-}$ peut se dismuter en $\mathrm{H}_{2} \mathrm{O}_{2}$, réagir avec $\mathrm{NO}^{\circ}$ pour former du peroxynitrite ONOO- ou avec des métaux de transition en formant des radicaux plus réactifs.

- Le radical hydroxyle $\mathrm{OH}^{\circ}$ est beaucoup plus réactif et peut attaquer la plupart des molécules biologiques et former des radicaux secondaires.

Ces ROS peuvent se transformer selon la réaction d'Haber-Weiss, par l'intervention de la superoxyde dismutase (SOD) et de métaux de transition comme $\mathrm{Fe}^{++}$ou $\mathrm{Cu}^{++}$libres, ou complexés (hème), qui sont des activateurs de l'oxydation.

- L'oxygène singulet ${ }^{1} \mathrm{O}_{2}$ peut être généré par des réactions photochimiques et chimiques (par exemple, la réaction de $\mathrm{H}_{2} \mathrm{O}_{2}$ avec $\mathrm{OCl}$ - produit ${ }^{1} \mathrm{O}_{2}$ ). ${ }^{1} \mathrm{O}_{2}$ est relativement réactif et peut former des peroxydes.

Certains radicaux sont formés par réaction d'électrons non appariés avec divers atomes (soufre, carbone) : ainsi les radicaux thiyls $\mathrm{RS}^{\circ}$ ont une forte réactivité et sont formés par oxydation de groupements thiols en présence de métaux de transition.

\section{Sources cellulaires de ROS}

Plusieurs systèmes enzymatiques capables de générer des ROS ont été identifiés dans les cellules vasculaires [2,5-8] :

- Les $N A D(P) H$ oxydases (NOX) sont présentes dans la paroi vasculaire et génèrent $\mathrm{O}_{2}^{\circ}$ - en utilisant NADH ou NADPH comme substrat. NOX existe sous différentes formes homologues Nox1, Nox2, et Nox 4, la forme Nox 2 étant bien exprimée dans les cellules vasculaires [5]. L'enzyme la mieux caractérisée est la NADPH oxydase leucocytaire qui est un complexe multimérique constitué par le cytochrome b558 (gp91phox et p22phox), et par des sous-unités régulatrices, p67phox, p47phox, p40phox et petites G-protéines rac-2 et rap-1A.

- La myéloperoxydase des cellules phagocytaires est une hème protéine qui peut générer $\mathrm{HOCl}$ (acide hypochloreux) en présence de $\mathrm{Cl}^{-}$, ou des radicaux tyrosyle en présence de tyrosine, ou former du peroxynitrite en présence de $\mathrm{NO}^{\circ}$.

- La xanthine oxydase/déshydrogénase peut générer $\mathrm{O}_{2}{ }^{0-}$ et $\mathrm{H}_{2} \mathrm{O}_{2}$ en utilisant comme substrat xanthine ou hypoxanthine et $\mathrm{NADH}$, et jouerait un rôle dans la production des ROS au cours de l'ischémie/reperfusion. - Les NO synthases (NOS) existent sous trois isoformes de NOS, neuronale (nNOS), inductible (iNOS), et endothéliale (eNOS), qui diffèrent par leur expression tissulaire et par leur dépendance vis-à-vis du $\mathrm{Ca}^{2+}$.

- Les lipoxygénases oxydent directement les AGPI. La 5-lipoxygénase oxyde l'acide arachidonique libre en hydroperoxyde (5-HPETE), précurseur des leucotriènes, puissants médiateurs de l'inflammation.

- La chaîne respiratoire mitochondriale génère $\mathrm{O}_{2}{ }^{\circ-}$ qui est ensuite converti en $\mathrm{H}_{2} \mathrm{O}_{2}$ par la SOD mitochondriale [9].

\section{Facteurs régulant le stress oxydatif}

Divers agents (physiques, chimiques, biologiques) sont capables de stimuler la formation des ROS par les cellules vasculaires. Les facteurs pro-inflammatoires (TNF $\alpha$, interleukines, LDLox et divers lipides), les toxines bactériennes (LPS), les AGE (advanced glycated end-products) augmentés en cas d'hyperglycémie induisent une production de ROS, de même que les facteurs hémodynamiques (flux sanguin, pression artérielle) et l'hypertension.

La stimulation des récepteurs à tyrosine kinase par leurs agonistes (EGF, PDGF) ou les récepteurs à 7 domaines transmembranaires/G-protéines (thrombine, bradykinine) induisent une production modérée de ROS impliquée dans l'effet mitogène et dans l'activation de voies de survie (Akt, NFkB) $[1,10,11]$.

\section{Les systèmes antioxydants}

Les systèmes antioxydants sont soit des molécules qui captent rapidement les ROS (anti-oxydants proprement dits), soit des systèmes enzymatiques qui catalysent la conversion des molécules pro-oxydantes [12, 13].

\section{Principaux systèmes enzymatiques antioxydants}

- Les superoxydes dismutases (SOD) existent sous trois types : SOD cytosolique à cuivre-zinc (CuZnSOD), mitochondriale à manganèse (MnSOD) et extracellulaire (CuZnSOD). Les SOD catalysent la dismutation de $\mathrm{O}_{2}{ }^{\circ-}$ en $\mathrm{H}_{2} \mathrm{O}_{2}$.

- La catalase dégrade spécifiquement $\mathrm{H}_{2} \mathrm{O}_{2}$ en $\mathrm{H}_{2} \mathrm{O}$ et $\mathrm{O}_{2}$. Dans les tissus de mammifères, la catalase serait localisée dans les peroxysomes.

- Les glutathion peroxydases (GPX). Quatre sont actuellement connues: la GPX classique (cGPX) est localisée dans le cytosol et les mitochondries, et utilise le glutathion réduit pour catalyser la réduction de $\mathrm{H}_{2} \mathrm{O}_{2}$ et des hydroperoxydes. La GI-GPX gastro-intestinale jouerait un rôle dans la réduction des peroxydes apportés par l'alimentation. La PHGPX catalyse la réduction des hydroperoxydes des AGPI des phospholipides. Le rôle de la P-GPX plasmatique est inconnu.

- Les hèmes oxygénases $(\mathrm{HO})$ existent sous deux formes, $\mathrm{HO}-1$ inductible (par le stress oxydatif), et $\mathrm{HO}-2$ constitutive.

\section{Les anti-oxydants}

Divers anti-oxydantss biosynthétisés par l'organisme, ou apportés par I'alimentation, régulent l'équilibre redox cellulaire [12-14].

- Glutathion. Le glutathion sous forme réduite (GSH) est un cofacteur des voies de détoxification, de régulation du potentiel redox et de réduction des thiols des protéines. Le GSH joue un rôle important dans la signalisation cellulaire et la régulation des grandes fonctions cellulaires (expression des gènes, apoptose).

- Vitamine C (acide ascorbique).

- Tocophérols. L'a-tocophérol ou vitamine E est capable de bloquer la propagation de la peroxydation en réduisant les peroxydes lipidiques en alcool.

- Composés phénoliques. Les polyphénols possèdent une activité antioxydante puissante, préviennent l'oxydation des LDL et ont un effet antiathérogène [15-17].

- Protéines de stockage ou de séquestration des métaux de transition. Les protéines liant les métaux (transferrine, lactoferrine, céruloplasmine...), l'albumine et l'haptoglobine diminuent leur pouvoir oxydatif.

D'autres agents présents dans les milieux biologiques (acide urique, bilirubine) de même que les HDL, peuvent exercer un effet antioxydatif. - Antioxydatifs de synthèse.

De nombreux antioxydatifs de synthèse sont utilisés comme additifs alimentaires : butylhydroxyanisole, butylhydroxytoluène, propylgallate. Ce sont des dérivés phénoliques qui agissent comme la vitamine $\mathrm{E}$.

\section{Effets cellulaires des ROS}

Les ROS agissent comme des seconds messagers qui interviennent dans plusieurs voies de signalisation mitogènes ou toxiques, en fonction de l'intensité du stress oxydatif généré.

\section{ROS et voies de prolifération/survie}

L'activation des récepteurs à tyrosine kinase (PDGFR, EGFR) ou de récepteurs couplés à des protéines $G$, induit une génération modérée de ROS impliqués dans la prolifération cellulaire. Les ROS activent la voie mitogène Ras/MAPK et l'activation d'AP-1. $\mathrm{H}_{2} \mathrm{O}_{2}$ activerait EGFR et PDGFR via une inhibition des protéines tyrosine phosphatases (PTPases). À côté de leur rôle dans la prolifération, les ROS sont impliqués dans l'activation de voies de stress et de survie dans les SMC ou les cellules endothéliales. 
L'angiotensine II, le VEGF ou les forces de cisaillement (shear stress) stimulent la $\mathrm{NAD}(\mathrm{P}) \mathrm{H}$ oxydase et $\mathrm{H}_{2} \mathrm{O}_{2}$.

Les ROS ont un effet biphasique sur le système ubiquitine/protéasome impliqué dans la dégradation des protéines modifiées par oxydation, activateur à dose modérée et inhibiteur à dose élevée, avec une implication dans l'apoptose. Les ROS activent le facteur de transcription redoxsensible NFkB qui joue un rôle majeur dans l'induction de gènes antiapoptotiques et pro-inflammatoires. Les mécanismes d'activation de NFkB par les ROS ne sont pas identifiés $[8-11,16]$.

\section{ROS et mort cellulaire}

Le déséquilibre de la balance prolifération/apoptose dépend de différents facteurs tels que la quantité de ROS générés, la durée du signal et l'état physiologique de la cellule. Les mécanismes impliqués dans la mort cellulaire font intervenir une inhibition des voies de survie antiapoptotiques ( $\mathrm{Pl}-3 \mathrm{~K} / \mathrm{Akt}$, du système ubiquitine/protéasome, NFkB), l'activation des caspases ainsi que des dysfonctions des systèmes de transport ioniques $[16,17]$.

\section{Caspases}

L'activation ROS dépendantes des caspases peut être la conséquence de I'activation de récepteurs à « death domain » (TNFR, Fas...), ou de l'altération directe des mitochondries par le stress oxydatif. Le détachement des cellules endothéliales (anoïkis) induit une forte génération de ROS et I'activation de caspases (bloquée par les antioxydatifs). Inversement, un taux élevé de ROS peut inhiber l'activité des caspases et bloquer l'apoptose des cellules qui meurent alors par nécrose.

\section{ROS, transport ionique et calcium intracellulaire}

Les ROS peuvent altérer les systèmes de transport ioniques (en oxydant les groupements thiols), en particulier les canaux calciques, les récepteurs aux IP3, les canaux $\mathrm{K}^{+}, \mathrm{Na}^{+}, \mathrm{Cl}^{-}$, les pompes $\mathrm{Ca}^{2+} /$ ATPase, $\mathrm{Na}^{+}, \mathrm{K}^{+}$-ATPase et $\mathrm{H}^{+}$-ATPase, de même que divers échangeurs ou transporteurs d'ions. Les ROS peuvent induire une dérégulation du calcium cytosolique, d'où altération des mitochondries et activation de caspases ou d'enzymes calcium dépendantes impliquées dans l'apoptose ou la nécrose (endonucléases, transglutaminases, calpaines).

En conclusion, les ROS ont des effets paradoxaux mitogènes ou toxiques, qui peuvent s'expliquer par des aspects quantitatifs (intensité et durée de production des ROS), topologiques (compartimentation subcellulaire), la présence d'autres facteurs redox (métaux de transition) et l'état redox de la cellule.

\section{Acides gras, ROS et pathologies vasculaires}

Les acides gras non estérifiés physiologiquement libérés durant la lipolyse sont chroniquement élevés dans des conditions pathologiques telles que l'obésité, le diabète insulino-résistant ou l'hypertension, qui sont des facteurs de risque majeurs dans l'athérosclérose. Les acides gras saturés seraient pro-athérogènes, alors que les acides gras insaturés comme I'acide oléique ou les acides gras polyinsaturés ( $n-3)$ sont considérés comme étant anti-athérogènes (World Health Organization, 1990) malgré des études controversées [18].

Le rôle des acides gras au cours de l'athérosclérose est actuellement mal identifié [18] : il pourrait résulter en partie d'une diminution du taux de LDL-cholestérol, ou de leur effet anti-inflammatoire (qui se traduit par une diminution de l'expression de molécules d'adhésion VCAM-1) [19], ou de leur effet antithrombotique (pour les acides gras n-3) [18]. Les mécanismes d'action sont complexes car les acides gras sont impliqués à différents stades de biologie cellulaire (membranes, métabolisme, production d'énergie, signalisation [20]). Au niveau cellulaire, les acides gras sont utilisés pour le renouvellement des membranes ou comme source d'énergie via la $\beta$-oxydation qui a lieu dans la mitochondrie. Les acides gras activent diverses voies de signalisation (PKC, MAPK) [20] ainsi que I'EGF récepteur (EGFR) via une modification de la fluidité membranaire, cet effet étant directement lié au degré d'insaturation des acides gras [21].

Les acides gras ont de multiples interactions avec la mitochondrie, et peuvent moduler les ROS mitochondriaux soit directement par la $\beta$-oxydation (qui active la chaîne respiratoire), soit via un effet découplant. Le mécanisme de découplage entre l'oxydation des substrats dans la chaîne respiratoire et la production d'énergie (ATP) pourrait impliquer un effet direct [22] ou au contraire indirect et passer par l'activation de protéines découplantes telles que UCP-2 [23, 24]. À dose élevée, les acides gras inhibent la chaîne respiratoire et peuvent augmenter la génération d'H2O2. Toutes ces observations ont été réalisées in vitro sur des mitochondries isolées et peu ou pas d'études ont été réalisées sur cellules intactes. Le rôle des acides gras sur la génération de ROS in vivo est donc peu connu.

Nous avons récemment mis en évidence que les acides gras modulent (inhibent) le stress oxydatif intracellulaire mitochondrial en stimulant une glutathion peroxydase (GPX), qui paraît activée via l'EGFR [25]. Nous faisons I'hypothèse que cet effet « antioxydatif » des acides gras pourrait intervenir dans leurs propriétés antiathérogènes.

\section{Effet des acides gras sur le stress oxydatif intracellulaire}

\section{Les acides gras bloquent le stress oxydatif mitochondrial}

Les mitochondries représentent une source majeure de ROS puisque 1 à $2 \%$ de l'oxygène consommé par les cellules serait converti en ion superoxyde $\mathrm{O} 2^{\circ}$ - au niveau des complexes I et III de la chaîne respiratoire mitochondriale. $\mathrm{O}^{\circ}$ - est ensuite transformé en $\mathrm{H}_{2} \mathrm{O}_{2}$ par la SOD mitochondriale $[26,27]$. La génération de ROS dans les mitochondries est stimulée par des inhibiteurs de la chaîne respiratoire, tels que l'antimycine (AM) qui inhibe le complexe III.

L'addition d'AM induit rapidement (en 10-15 minutes) une production importante de ROS dans les cellules endothéliales-like ECV304 et les CML humaines. La co-incubation avec l'acide oléique bloque complètement la génération de ROS par I'antimycine (figure 1). Cet effet dépend du rapport acide gras/albumine dans le milieu de culture cellulaire (effet maximal pour un ratio 1/1), mais ne dépend pas de la longueur de chaîne de l'acide gras ni du degré d'insaturation. La suite de ces travaux a été réalisée avec l'acide oléique qui est peu sensible aux phénomènes d'oxydation (qui peuvent altérer les AGPI, et donc modifier l'interprétation des résultats).

Comme les acides gras peuvent se comporter comme des agents découplants sur mitochondries isolées [27-29], et que les agents découplants diminuent le potentiel de membrane mitochondrial, $\Delta \psi_{m}$ et la génération de ROS [29,30], nous avons étudié l'effet de l'acide oléique sur le potentiel de membrane. Cependant, dans nos conditions expérimentales, l'acide oléique n'induit aucune modification du $\Delta \Psi_{\mathrm{m}}$ dans les cellules intactes. De plus, les agents découplants classiques tels que le carbonyl cyanide $m$-chlorophenylhydrazone, ne bloquent pas la génération de ROS et peuvent également générer des ROS par eux-mêmes. Ces résultats suggèrent que l'inhibition des ROS par l'acide oléique n'est pas due à un effet découplant. Une hypothèse est que les acides gras pourraient soit induire l'expression de systèmes anti-oxydants, soit stimuler leur activité. Or, une surexpression de systèmes de défenses antioxydantes cellulaires paraît peu probable car le cycloheximide (inhibiteur de synthèse des protéines) ne réverse pas l'effet protecteur des acides gras. Nous avons recherché l'effet de l'acide oléique sur les enzymes antioxydatifes cellulaires. 


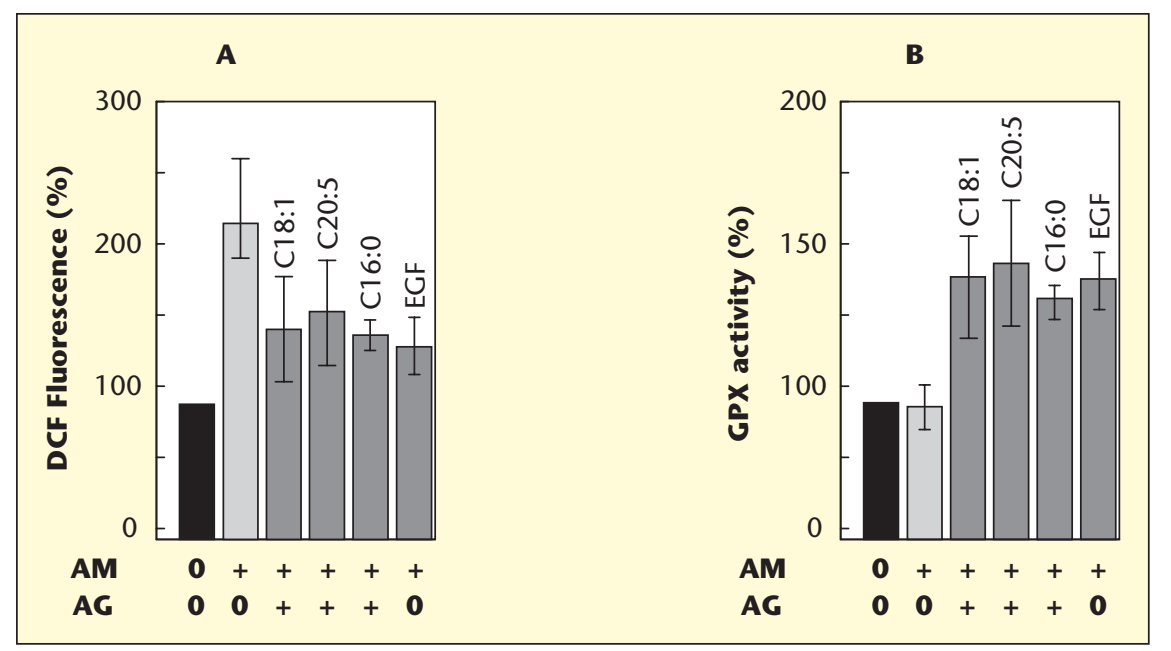

Figure 1. Effet des acides gras sur le stress oxydatif mitochondrial.

A. Effet inhibiteur des acides gras sur la génération de ROS mitochondriaux. Les cellules ECV304 sont incubées en milieu RPMI 1640 contenant $50 \mu M$ de serum albumine bovine (fatty acid free), et $5 \mu \mathrm{M}$ de sonde DCFDA-AM. Après 30 minutes de marquage avec la sonde, les cellules sont stimulées pendant 15 minutes avec $10 \mu \mathrm{M}$ d'antimycine A (AM), en présence ou en absence de $50 \mu \mathrm{M}$ des acides gras (AG), ou $10 \mathrm{nM}$ EGF. Les cellules sont lavées et scrappées en PBS, et la fluorescence des cellules est mesurée (longueur d'onde excitation $490 \mathrm{~nm}$, émission, $525 \mathrm{~nm}$ ). Les protéines cellulaires sont mesurées par la technique de Bradford [25]. Les résultats sont exprimés en \% du contrôle non stimulé, et sont la moyenne de 10 expériences séparées. B. Activation de la GPX par les acides gras. Les ECV304 sont incubées en présence d'antimycine et d'acides gras comme décrit en 1 A. L'activité enzymatique de la GPX est mesurée comme décrit en [25]. Les résultats sont exprimés en \% de l'activité du contrôle.

\section{L'acide oléique stimule l'activation de la GPX}

Nous avons étudié l'effet de l'acide oléique sur les activités catalase et GPX qui dégradent $\mathrm{H} 2 \mathrm{O} 2$ dans la cellule. L'incubation des cellules avec l'acide oléique n'induit aucune modification de la catalase, alors que I'activité GPX est rapidement augmentée en présence d'acide gras (figure 1). De plus, l'addition de mercaptosuccinate, un inhibiteur de GPX, inhibe l'activation de GPX observée en présence d'acide oléique et réverse l'effet inhibiteur sur la génération de ROS par AM [25]. Les GPX sont régulées par divers stimuli physiologiques ou pathologiques. Leur expression diminue avec le déficit en sélénium, le stress oxydatif ou la formation d'adduits par glycation ou glycoxydation, et est augmentée par le shear stress, I'ischémie, l'hypertension, les endotoxines et l'obésité $[31,32]$. Dans nos conditions expérimentales, les acides gras n'induisent pas de surexpression de la GPX, puisque le cycloheximide n'inhibe pas l'effet activateur de l'acide oléique. Par ailleurs, l'acide oléique n'active pas la GPX in vitro, ce qui suggère que l'activation de GPX dans la cellule vivante résulterait plutôt d'une signalisation activée par les acides gras [25].

\section{L'activation de la GPX par l'acide oléique dépend de l'EGF récepteur}

Les acides gras activent de nombreuses voies de signalisation, telles que la PKC, les MAP kinases, la PI3 kinase et l'EGFR $[20,21]$. La phosphorylation et l'activation de l'EGFR par les acides gras est due (en partie) à une modification de la fluidité membranaire [21]. D'autre part, l'EGF peut réguler la génération de ROS, qui sont impliqués dans la signalisation de I'EGFR [21, 33]. Nous avons étudié l'implication de l'EGFR dans I'effet protecteur de I'acide oléique et l'activation de la GPX, par plusieurs approches : i) I'utilisation d'un inhibiteur spécifique de l'EGFR, l'AG1478, ii) l'effet direct de l'EGF, iii) l'effet de l'acide oléique sur des cellules dominant négatif pour l'EGFR. Les résultats obtenus montrent que l'effet protecteur de l'acide oléique sur les ROS mitochondriaux de même que I'activation de GPX dépendent de I'EGFR et ces résultats sont reproduits par l'EGF (figures 1 et 2) et [25].

\section{Les acides gras inhibent la formation de 8oxodG induit par le stress oxydatif}

Le stress oxydatif intracellulaire induit des lésions de I'ADN d'abord mitochondrial puis nucléaire [34]. Nous avons testé l'effet de l'acide oléique sur une conséquence majeure du stress oxydatif mitochondrial, soit la formation de lésions de l'ADN (formation des 8-0xo-7,8-dihydro2'-deoxyguanosine, 8oxodG).

Les 8 oxodG sont mesurés sur mitochondries isolées exposées à temps variables à de l'antimycine $\mathrm{A}$ (15 et 30 minutes), en présence ou en absence d'acide oléique. Comme rapporté dans la figure $2 A$, l'antimycine $A$ induit une augmentation significative des $80 x o d G$ ( 200 à $250 \%$ du contrôle) (les deux paramètres étant mesurés en HPLC). L'acide oléique bloque la formation des 8 oxodG ( $120 \%$ du contrôle).

Dans un second temps, l'effet de l'acide oléique sur la formation de $80 x o d G$ a été étudié in situ sur des cellules ECV304 soumises à un stress oxydatif soit mitochondrial (antimycine $\mathrm{A})$, ou plus général (H2O2). Les cellules sont stimulées pendant 20 minutes par $\mathrm{H} 2 \mathrm{O} 2$ ou antimycine, en présence ou en absence d'acide oléique et d'albumine. Les résultats sont comparés à ceux obtenus en remplaçant l'acide oléique par l'EGF. Les 8oxodG sont quantifiés par technique Elisa (Trevigen) et par immunocytochimie couplée à une observation en microscopie confocale.

Les résultats rapportés dans la figure $2 B$ sont corrélés à ceux observés sur des mitochondries isolées et confirment que l'acide oléique, dans les conditions où il bloque le stress oxydatif mitochondrial, protège contre la formation de lésions 8oxodG induites par l'antimycine A. L'effet protecteur de l'acide oléique implique le récepteur à l'EGF, comme le suggère l'effet protecteur très marqué de l'EGF $(10 \mathrm{nM})$ (figures $2 B$ et $C$ ). Weinbrenner et al. [35] ont rapporté récemment que l'absorption d'huile d'olive enrichie en polyphénols diminue l'oxydabilité des LDL et la formation de 8oxodG mitochondrial et urinaire. Notre étude montre que l'acide oléique peut exercer un effet protecteur (antioxydatif-like) sur la formation de lésions dans I'ADN.

En conclusion, ces résultats mettent en évidence des propriétés nouvelles des acides gras, contre le stress oxydatif et la formation de lésions dans I'ADN. Le mécanisme d'activation de la GPX via l'activation de I'EGFR, 


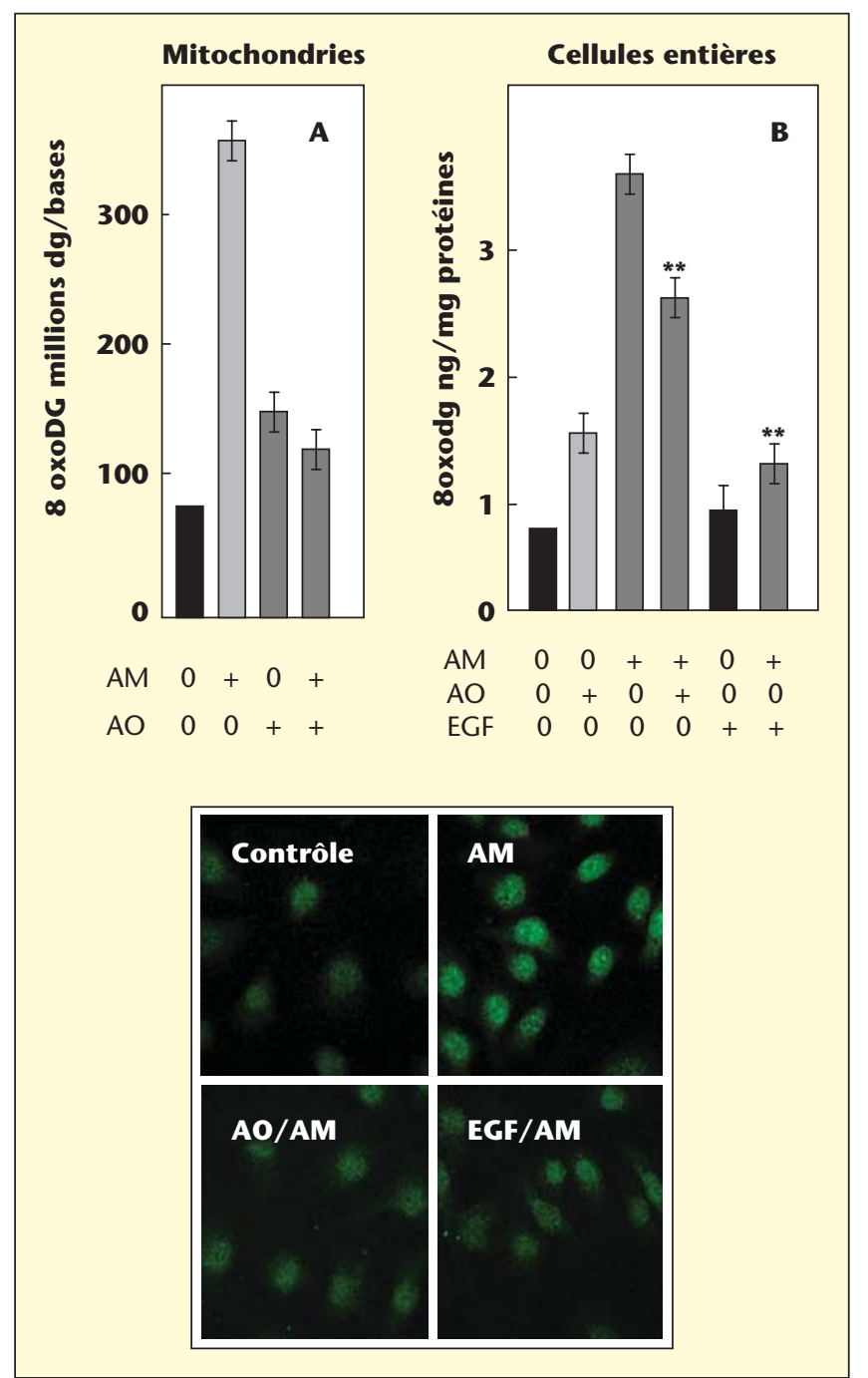

Figure 2. Effet protecteur des acides gras sur la formation des $80 x o d \mathrm{G}$ mitochondriaux. A. Effet sur mitochondries isolées. Les mitochondries de foie de rat en suspension dans du milieu RPMI contenant $50 \mu \mathrm{M}$ de sérum albumine bovien, sont stimulées pendant 15 min. par l'antimycine (AM, $10 \mu \mathrm{M})$, en présence ou en absence d'acide oléique (AO). L'ADN mitochondrial est purifié et la présence de 80xodG est mesurée en HPLC, comme décrit en [37]. B. Effet sur cellules entières. Les ECV304 sont stimulées par I'Antimycine A et l'acide oléique ou l'EGF comme décrit en Figure 1. La formation de 8oxodG est évaluée par test Elisa (Trevigen). Les résultats sont exprimés en \% du contrôle non stimulé. C. Étude de la formation des 8oxodG en immunocytochimie (anticorps ant 80xodG, Trevigen), dans les cellules ECV304 stimulées par $100 \mu \mathrm{M}$ $d^{\prime} \mathrm{H}_{2} \mathrm{O}_{2}$, en présence ou en absence d'acide oléique $(A O, 50 \mu \mathrm{M})$ ou d'EGF $(10 \mathrm{nM})$.

reste à démontrer mais pourrait constituer un mécanisme de feedback permettant de diminuer à la fois la quantité de $\mathrm{H} 2 \mathrm{O} 2$ et la phosphorylation de l'EGFR, et donc de réguler la signalisation EGFR dépendante et les défenses cellulaires contre le stress oxydatif.

Sur un plan physiopathologique, l'apoptose des cellules vasculaires induite par les LDL oxydées ou le stress oxydatif est impliquée dans la rupture de plaque et la thrombose. De plus, les cassures de I'ADN mitochondrial sous l'effet d'un stress oxydatif pourraient représenter une étape très précoce du déclenchement de l'apoptose et du remodelage de la plaque. Par ailleurs, I'activation de l'EGFR par les LDL oxydées ou $\mathrm{H} 2 \mathrm{O} 2$ serait impliquée dans la survie des cellules vasculaires via l'activation de la voie de survie PI3 kinase/Akt [36], mais pourrait également agir en renforçant les défenses anti-oxydatifes (GPX). Les acides gras pourraient (via l'EGFR) inhiber l'apoptose induite par des agents pro-oxydants ou par les LDL oxydées [36, 37], et donc participer à la stabilisation de la plaque d'athérosclérose.

Remerciements. Ce travail a été réalisé avec I'aide de I'Inserm, de I'Université Paul Sabatier, Toulouse et du GLN.

\section{RÉFÉRENCES}

1. DHALLA NS, TEMSAH RM, NETTICADAN T. Role of oxidative stress in cardiovascular diseases. J Hypertens $2000 ; 18: 655-73$.

2. PATEL RP, MOELLERING D, MURPHY-ULLRICH J, JO H, BECKMAN JS, DARLEY-USMAR VM. Cell signaling by reactive nitrogen and oxygen species in atherosclerosis. Free Radic Biol Med $2000 ; 28:$ 1780-94.

3. HALLIWELL B, GUTTERIDGE JMC. Free Radicals in Biology and Medicine. Oxford: Clarendon Press, 1989.

4. BOVERIS A, OSHINO N, CHANCE B. The cellular production of hydrogen peroxide. Biochem / $1972 ; 128: 617-30$.

5. GRIENDLING KK, SORESCU D, USHIO-FUKAI M. NAD(P)H oxidase : Role in cardiovascular biology and diseases. Circ Res $2000 ; 86: 494-501$.

6. CHANCE B, SIES H, BOVERIS A. Hydroperoxide metabolism in mammalian organs. Physiol Rev $1979 ; 59:$ 527-605.

7. THANNICKAL V], FANBURG BL. Reactive oxygen species in cell signaling. Am J Physiol Lung Cell Mol Physiol 2000 ; 279 : L1005-L1028.

8. WOLIN MS. Interactions of oxidants with vascular signalings systems. Arterioscler Thromb Vasc Biol $2000 ; 20$ : 1430-42.

9. TURRENS JF. Superoxide production by the mitochondrial respiratory chain. Biosci Rep 1997 ; 17 : 3-8.

10. HENSLEY K, ROBINSON KA, GABBITA SP, SALSMAN S, FLOYD RA. Reactive oxygen species, cell signaling, and cell injury. Free Radicals Biol Med 2000 ; $28: 1456-62$

11. LEONARDUZZI G, ARKAN MC, BASAGA H, CHIARPOTTO E, SEVANIAN A, POLI G. Lipid oxidation products in cell signaling. Free Radicals Biol Med $2000 ; 28: 1370-8$.

12. WASSMANN S, WASSMANN K, NICKENIG G. Modulation of oxidant and antioxidant enzyme expression and function in vascular cells. Hypertension $2004 ; 44: 381-6$

13. SCHULZ E, ANTER E, KEANEY JP. Oxidative stress, antioxidants, and endothelial function. Curr Med Chem $2004 ; 11$ : 1093-104.

14. ABRAHAM NG, KAPPAS A. Heme oxygenase and the cardiovascular-renal system. Free Radic Biol Med $2005 ; 39: 1$ :-25.

15. VIOLI F, CANGEMI R. Antioxidants and cardiovascular disease. Curr Opin Investig Drugs $2005 ; 6: 895-900$.

16. SALVAYRE R, AUGE N, NEGRE-SALVAYRE A. Rôle de I'oxydation dans la genèse et la progression de l'athérosclérose. In : Toussaint JF, Jacob MP, Lagrost L, Chapman J, eds. L'athérosclérose. Paris : Masson, 2003 : 269-90.

17. SALVAYRE R, NEGRE-SALVAYRE A. Aspects cellulaires et mécanismes de I'apoptose dans l'athérosclérose. In: Toussaint JF, Jacob MP, Lagrost L, Chapman J, eds. L'athérosclérose. Paris : Masson, 2003 : 313-32.

18. RAVNSKOV U. The questionable role of saturated and polyunsaturated fatty acids in cardiovascular disease. / Clin Epidemiol 1998 ; 51 : 443-60.

19. DE CATERINA R, CYBULSKY MI, CLINTON SK, GIMBRONE JR. MA, LIBBY P. The omega-3 fatty acid docosahexaenoate reduces cytokine-induced expression of proatherogenic and proinflammatory proteins in human endothelial cells. Arterioscler Thromb 1994 ; 14 : 1829-36. 
20. BANDYOPADHYAY GK, HWANG S, IMAGAWA W, NANDI S. Role of polyunsaturated fatty acids as signal transducers : amplification of signals from growth factor receptors by fatty acids in mammary epithelial cells. Prostaglandins Leukotr Essent Fatty Acids $1993 ; 48$ : 71-8.

21. VACARESSE N, LAJOIE-MAZENCI, AUGE N, et al. Activation of epithelial growth factor receptor pathway by unsaturated fatty acids. Circ Res 1999 ; $85: 892-9$.

22. KORSHUNOV SS, KORKINA OV, RUUGE EK, SKULACHEV VP, STARKOV AA. Fatty acids as natural uncouplers preventing generation of $\mathrm{O}_{2}{ }^{-}$and $\mathrm{H}_{2} \mathrm{O}_{2}$ by mitochondria in the resting state. FEBS Lett $1998 ; 435: 215-8$.

23. CHEVILLOTTE E, RIEUSSET J, ROQUES M, DESAGE M, VIDAL H. The regulation of uncoupling protein-2 gene expression by omega- 6 polyunsaturated fatty acids in human skeletal muscle cells involves multiple pathways, including the nuclear receptor peroxisome proliferator-activated receptor beta. J Biol Chem 2001 ; 276 : 10853-60.

24. NEGRE-SALVAYRE A, HIRTZ C, CARRERA G, et al. A role for uncoupling protein-2 as a regulator of mitochondrial hydrogen peroxide generation. FASEB / $1997 ; 11: 809-15$.

25. DUVAL C, AUGE N, FRISACH MF, CASTEILLA L, SALVAYRE R, NEGRESALVAYRE A. Mitochondrial oxidative stress is modulated by oleic acid via an epidermal growth factor receptor-dependent activation of glutathione peroxidase. Biochem / 2002 ; 367(Pt 3) : 889-94.

26. BOVERISA, CHANCE B. The mitochondrial generation of hydrogen peroxide. General properties and effect of hyperbaric oxygen. Biochem J $1973 ; 134: 707-16$.

27. LOSCHEN G, AZZI A, RICHTER C, FLOHE L. Superoxide radicals as precursors of mitochondrial hydrogen peroxide. FEBS Lett $1974 ; 42$ : 68-72.
28. WOJTCZAK L, SCHONFELD P. Effect of fatty acids on energy coupling processes in mitochondria. Biochim Biophys Acta $1993 ; 1183: 41-57$.

29. HERMESH O, KALDERON B, BAR-TANA J. Mitochondria uncoupling by a long chain fatty acyl analogue. J Biol Chem 1998 ; 273 : 3937-42.

30. SKULACHEV VP. Uncoupling : new approaches to an old problem of bioenergetics. Biochim Biophys Acta $1998 ; 1363$ : 100-24.

31. ARTHUR JR. The glutathione peroxidases. Cell Mol Life Sci $2000 ; 57: 1825$ 35 .

32. BRIGELIUS-FLOHE R. Tissue-specific functions of individual glutathione peroxidases. Free Radicals Biol Med $1999 ; 27$ : 951-65.

33. PRICE LT, CHEN Y, FRANK L. Epidermal growth factor increases antioxidant enzyme and surfactant system development during hyperoxia and protects fetal rat lungs in vitro from hyperoxic toxicity. Pediatr Res $1993 ; 34$ : 577-85.

34. BECKMAN KB, AMES BN. Endogenous oxidative damage of mtDNA. Mutat Res $1999 ; 424: 51-8$.

35. WEINBRENNER T, FITO M, DE LA TORRE R. Olive oils high in phenolic compounds modulate oxidative/antioxidative status in men. J Nutr 2004 ; $134: 2314-21$.

36. AUGE N, GARCIA V, MAUPAS-SCHWALM F, LEVADE T, SALVAYRE R, NEGRE-SALVAYRE A. Oxidized LDL-induced smooth muscle cell proliferation involves the EGF receptor/PI-3 kinase/Akt and the sphingolipid signaling pathways. Arterioscler Thromb Vasc Biol 2002 ; 22 : 1990.

37. SASTRE J, PALLARDO FV, VINA J. The role of mitochondrial oxidative stress in aging. Free Radic Biol Med $2003 ; 35$ : 1-8. 